Q. GIG Bwrdd lechyd Cwm Taf NHS Health Board

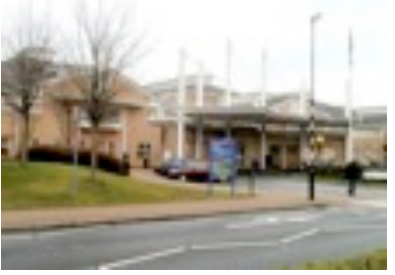

\title{
Liraglutide as an effective therapeutic agent in a patient with Prader-Willi Syndrome and type 2 diabetes.
}

Witczak JK, Owen PJD

Department of Diabetes and Endocrinology, Royal Glamorgan Hospital, Cwm Taf Health Board, Llantrisant

\section{Introduction}

Prader Willi syndrome is a genetic disorder associated with learning disability, hyperphagia and obesity which leads to early development of obesity related complications. The weight and appetite management remains a challenge with not much success with appetite suppressant drugs and bariatric surgery.

GLP-1 receptor analogues appear to be a promising alternative given their effect on appetite control, weight loss and HbAlc and were shown to decrease the levels of plasma ghrelin in PWS patients.
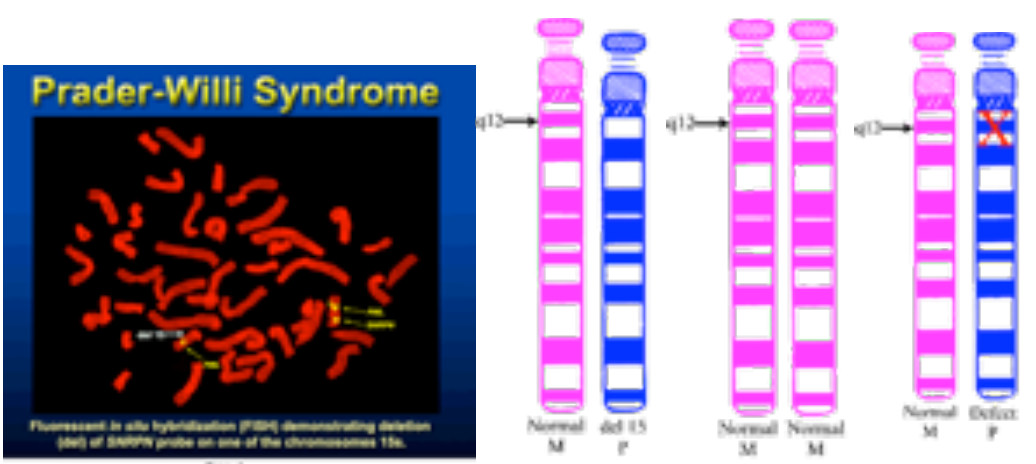

Case history

A 25 year old white female with PWS presented to diabetic clinic with newly diagnosed type 2 diabetes. She was delivered at 35 weeks gestation (weight $2.63 \mathrm{~kg}$ ) but at 4 weeks became unwell and hypotonic which raised the clinical suspicion of PWS. The subsequent molecular analysis revealed kariotype 46, XX, del(15)(q11.2q11.2).

At the time of clinical manifestation of her diabetes she already had an extensive list of obesity-related comorbidities: lymphoedema, previous PE, gout and previous cholecystectomy. She struggled to manage her weight with dietary interventions. She was initially treated with metformin and gliclazide which failed to achieve the target $\mathrm{HbAlc}$ nor improved her weight. A decision was made to give her a trial of liraglutide- $0.6 \mathrm{mg}$ daily subsequently titrated to $1.2 \mathrm{mg}$ daily with metformin $500 \mathrm{mg}$ bd being continued.

\section{Results}

In October 2011 when liraglutide was commenced patient's weight was 148kg (BMI 65) with HbAlc of $64 \mathrm{mmol} / \mathrm{mol}$. By May 2012 the weight has improved to $145 \mathrm{~kg}$ and $\mathrm{HbAlc}$ to $51 \mathrm{mmol} / \mathrm{mol}$. One year later her weight has further improved to 139kg (BMI 61) and her glycaemic control remains within target $(54 \mathrm{mmol} / \mathrm{mol})$.

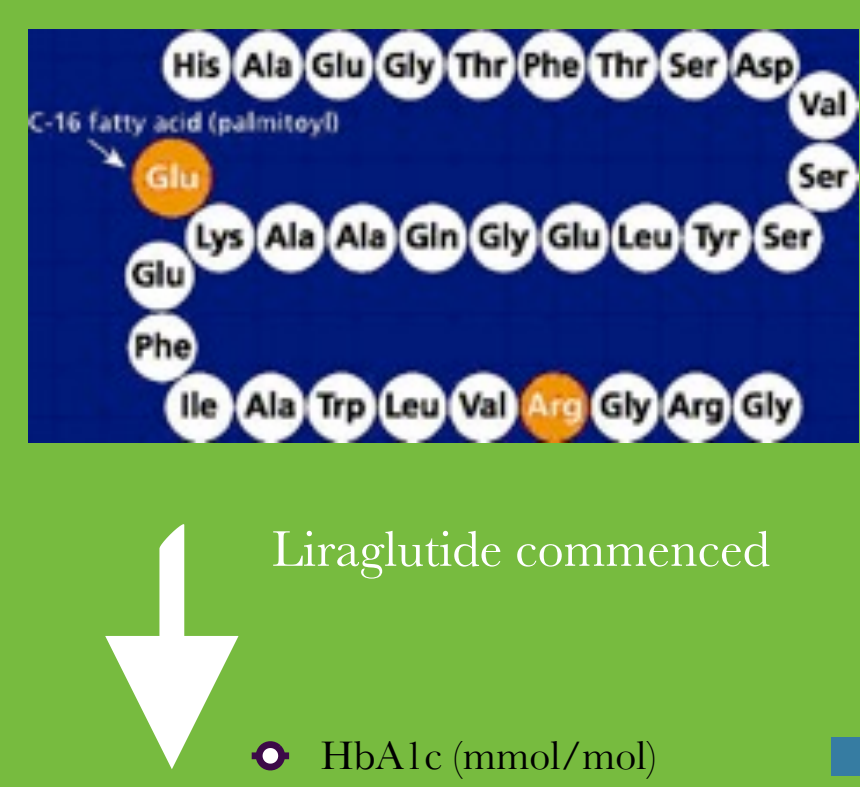

Weight $(\mathrm{kg})$

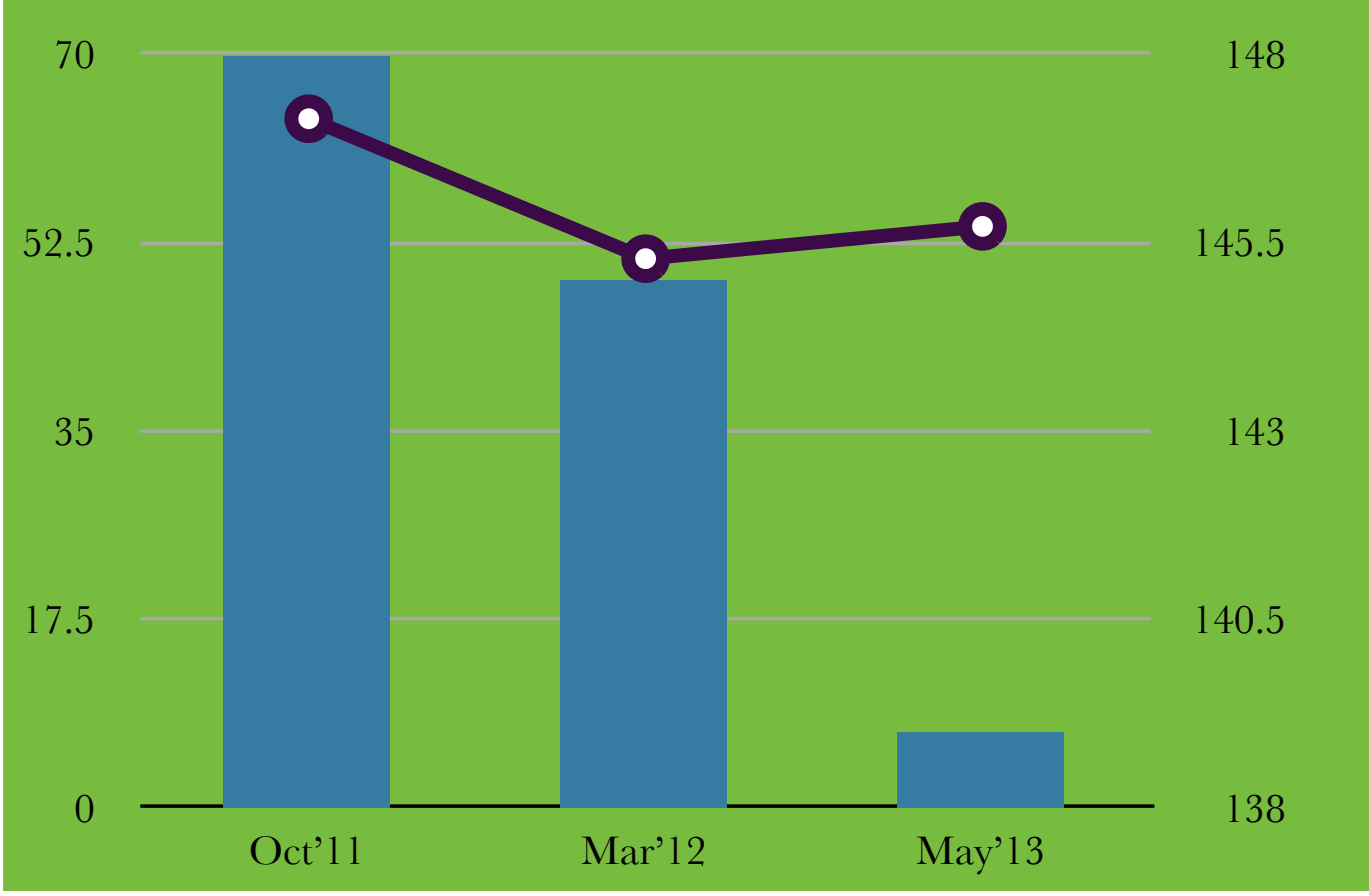

Conclusions

Although there are only few cases reported on GLP1RA use in PWS patients the results are encouraging given the potential possibility of extending the use of these agents for obesity management in future. 